\title{
OTROS MODELOS DE EMPRESA EN LA ECONOMÍA SOLIDARIA: ENTRE LA RETÓRICA Y LA PRÁCTICA
}

\author{
Juan Carlos Pérez-Mendiguren Castresana \\ Departamento de Organización de Empresas, UPV/EHU. \\ HEGOA-Instituto de Estudios sobre Desarrollo y Cooperación Internacional, UPV/EHU \\ ENEKOITZ ETXEZARRETA ETXARRI \\ Departamento de Economía Aplicada I, UPV/EHU. \\ GEZKI-Instituto de Derecho Cooperativo y Economía Social, UPV/EHU
}

DOI: 10.1387/lan-harremanak.16110

\section{ABSTRACT}

Este articulo propone una reflexión critica sobre la factibilidad de construir otros modelos de empresa en el marco de lo que se ha venido a denominar Economía Solidaria. El artículo presenta un recorrido teórico y empirico por los elementos principales que construyen la lógica organizativa de las organizaciones de Economía Solidaria, y apoyándose en la evidencia empirica existente ofrece una discusión de los principales retos y contradicciones a los que se enfrentan este tipo de organizaciones en su camino hacia la construcción de otra economía.

Palabras clave: economía solidaria; empresas solidarias; factor C; desacople organizacional.

This article offers a critical enquiry on the feasibility of creating "different business models» based on the premises of what has been named as the Solidarity Economy. The article reviews from a theoretical and empirical perspective what constitute the building blocks of the Solidarity Economy Organizations, and dwelling on the 
existing empirical evidence, it looks into the challenges and trade-offs faced by these organizations in their way towards the construction of "another economy"

Keywords: solidarity economy; solidarity economy businesses; factor C; organizational decoupling.

Artikulu honek "bestelako enpresa ereduen" gaineko gogoeta kritikoa proposatzen du, Ekonomia Solidarioa deitu izan denaren testuinguruaren baitan. Artikuluak, beraz, Ekonomia Solidarioa osatzen duten erakundeen antolakuntza-logikaren osagai teoriko eta praktiko nagusiei erreparatu die, eta ebidentzia enpirikoan oinarri hartuz, "bestelako ekonomia" sortze bidean erakunde horiek aurrean dituzten erronka eta kontraesanen inguruko eztabaida xaxatu nahi du.

Hitz gakoak: ekonomia solidarioa; enpresa solidarioak; C-Faktorea; antolakuntza banantzea. 


\section{Introducción}

Este artículo propone una reflexión crítica sobre la factibilidad de construir en la teoría y en la práctica otros modelos de empresa en el marco de lo que se ha venido a denominar Economía Solidaria, concepto que se está consolidando como una referencia habitual en los debates sobre la construcción de alternativas económicas al modelo actual, tanto a nivel internacional, como en contextos nacionales, regionales y locales.

Como hemos argumentado en anteriores trabajos (Pérez de Mendiguren y Etxezarreta, 2015a, 2015b; Pérez de Mendiguren et. al, 2009; Pérez de Mendiguren, 2014), con el término Economía Solidaria se hace referencia a un conjunto heterogéneo de enfoques teóricos, realidades socio-económicas y prácticas empresariales que desde el último cuarto del siglo xx vienen desarrollando un creciente sentido de pertenencia a una forma diferente de entender el papel de la economía y los procesos económicos en las sociedades contemporáneas. Frente a la lógica del capital, la mercantilización creciente de las esferas públicas y privadas y la búsqueda de máximo beneficio, la Economía Solidaria persigue construir relaciones de producción, distribución, consumo y financiación basadas en la justicia, la cooperación, la reciprocidad y la ayuda mutua. Frente al capital y su acumulación, la Economía Solidaria aboga por situar a las personas y su trabajo en el centro del sistema económico, otorgando a los mercados un papel instrumental siempre al servicio del bienestar de todas las personas y de la reproducción de la vida en el planeta (Pérez de Mendiguren et al., 2009).

Además de obvios vínculos históricos y conceptuales con la Economía Social $^{1}$, la Economía Solidaria reúne en su cuerpo teórico conceptos e ideas con

\footnotetext{
1 A diferencia del anterior, el concepto de Economía Social goza de una amplia tradición en la literatura académica (Monzón y Chaves, 2012, 2011; Monzón, 2003; Chaves et. al, 2003; Etxezarreta y Morandeira, 2012). Las organizaciones clásicas de la Economía Social (cooperativas, mutualidades y asociaciones) comparten compromiso fundacional con una serie de valores y principios de actuación que estructuran su lógica organizativa y su actividad empresarial en base a los siguientes elementos:

a) una clara preeminencia de las personas sobre el capital (tanto en la toma de decisiones como en el reparto del excedente);
} 
origen en Latinoamérica y Europa (Guerra 2011, 2010; Da Ros, 2007) y se refiere a un conjunto heterogéneo de prácticas que se manifiestan en todas las esferas del proceso económico, (i.e. producción, distribución, financiación y consumo) que buscan garantizar la seguridad de los medios de vida de las personas y democratizar la economía y los procesos económicos (Coraggio 2011; Laville y García, 2009).

No existe una definición comúnmente aceptada sobre el concepto. Sin embargo, y a pesar de las diferencias, existe en la todavía escasa literatura sobre el tema cierto consenso en relación el carácter multidimensional del concepto (Coraggio, 2012, 2011; Guerra 2013, 2010; Martínez y Álvarez, 2008), que recogería al menos tres dimensiones complementarias.

Cabe identificar en primer lugar una dimensión teórica interesada en construir un paradigma alternativo sobre la economía, que parte de la crítica al paradigma convencional. Los referentes teóricos sobre los que sustentar esta visión alternativa son de naturaleza diversa e incluyen tanto contribuciones de la antropología y la sociología critica como de las diferentes corrientes de la economía crítica europea y latinoamericana (Laville, 2009, 2004; Martínez y Álvarez, 2008; Hintze 2010, Coraggio, 2009).

La segunda dimensión se refiere a la idea de Economía Solidaria como una propuesta política de transformación social hacia un modelo socio-económico alternativo al derivado de la lógica capitalista. Los mecanismos de transformación y las dinámicas que adoptan estas propuestas de superación del capitalismo, no se presentan como modelos cerrados, sino como tránsitos hacia horizontes posibles, en los que los procesos económicos estén al servicio de la reproducción de la vida y sometidos a procesos democráticos participativos e inclusivos. En función de los autores, estos procesos en construcción vinculan la Economía Solidaria con la construcción de una "Economía Plural» (Laville, 2010), "Economía del Trabajo» (Coraggio, 2007), "Economía Matrística» (Arruda, 2005, 2004) o alguna modificación o derivado de los anteriores (Guerra, 2011).

La tercera dimensión identifica la Economía Solidaria con un tipo específico de empresa basada en la democracia, la autogestión y el empresariado colectivo (Martínez y Álvarez, 2008), y que busca funcionar con una lógica diferente a las de las organizaciones mercantiles tradicionales. En este sentido, muchas de las organizaciones o empresas de que se identifican como de Economía Solidaria, adoptan discursos y formas institucionales típicas de la Economía Social clásica

b) la apuesta por la autonomía y democracia en la gestión;

c) la solidaridad (interna y externa) y;

d) la prioridad del servicio a sus miembros y a la comunidad por encima de la consecución de beneficios. 
aunque no exclusivamente 2 . Desde el punto de vista geográfico, están localizadas tanto en países y regiones del llamado Norte Global como en países del Sur Glo$b a l$, y su actividad puede estar relacionada con cualquiera de las esferas del proceso económico (Martínez y Álvarez, 2008; Askunze, 2013, 2007).

Por lo tanto si bien el significante Economía Solidaria alude a significados que trascienden el ámbito puramente organizativo-empresarial, es indudable que mucho del interés que el término suscita se debe a su relación directa con procesos y organizaciones que tratan de construir otra economía partiendo de otra forma de ser y hacer empresa. Como hemos argumentado en anteriores trabajos (Pérez de Mendiguren, 2014, 2013) esta relación explícita entre el proyecto político post-capitalista y las dinámicas organizativas de corte empresarial permite distinguir entre este concepto y otros relacionados como los de Empresa Social (o Emprendimiento Social) que, si bien comparten la preocupación por la generación de modelos alternativos de empresa, no se vinculan tan claramente a la necesidad de trascender el modelo económico capitalista ${ }^{3}$.

Esta cuestión es relevante para entender por qué este artículo (en el contexto de este número especial) se interesa particularmente en los procesos y modelos de empresa identificados con la Economía Solidaria, aunque en algunos casos haga referencia a los aprendizajes derivados de los otros modelos de empresa vinculados a los conceptos de Empresa Social, Emprendimiento Social y/o Economía Social.

En cuanto a las lógicas discursiva y metodológica que guían este trabajo, ambas ofrecen una doble vertiente. Con respecto a los elementos discursivos, el artículo comienza con un breve repaso a la realidad, y al potencial de la Economía Solidaria en la generación de nuevos modelos de organización/empresa con vocación de contribuir a la construcción de dinámicas transformadoras del sistema. Sin embargo, tratamos a su vez de recoger la preocupación de Utting (2015) que alerta sobre la tendencia de la literatura sobre el tema a generar visiones excesivamente románticas sobre las experiencias de Economía Solidaría, que ocultan la contradicciones y tensiones que se experimentan, en particular cuando se trata de aumentar la escala de operación y capacidad de incidencia en la sociedad de estos emprendimientos. En el ámbito de la Economía Social, esta

2 La estrecha relación entre los conceptos de Economía Social y Economía Solidaria, así como los debates asociados a la generalización del término Economía Social y Solidaria, ha sido ampliamente abordada por los autores de este artículo en trabajos anteriores. Ver en particular: Pérez de Mendiguren y Etxezarreta, 2015; Pérez de Mendiguren, 2014.

3 En el caso del concepto de Economía Social, la distinción no está tan clara ya que la posición varía en función de si se mira al proyecto originario de la Economía Social, o al resultado de la progresiva institucionalización que este concepto ha experimentado a lo largo del siglo xx. Para una discusión detallada de este extremo ver también Laville y García (2009); Moulaert y Ailenei (2005); Laville (2004). 
preocupación ha sido también señalada por Heras (2014) en un reciente artículo sobre la experiencia cooperativa de Mondragon, en el que apunta a la existencia de una tradición académica clásica ${ }^{4}$ que no toma en consideración las paradojas y tensiones generadas por la experiencia, y que tiende a presentarla como un caso de éxito gerencial, elevado a la categoría de mito monolítico, ejemplo de aplicación de métodos democráticos y participativos a la gestión de organizaciones empresariales (Heras, 2014: 647).

En este sentido, nuestro trabajo quiere huir de esta tendencia a los relatos míticos, introduciendo en la discusión aquellas tensiones, contradicciones y disyuntivas que se generan en el marco de organizaciones que quieren generar alternativas al sistema operando desde el interior del mismo, y con las herramientas de gerencia y administración que han sido generadas para atender a su propia lógica de reproducción.

Por último, y refiriéndonos ya a la lógica metodológica utilizada en el artículo, si bien estamos ante una reflexión fundamentalmente teórica, hemos tratado de enriquecer la argumentación proponiendo un viaje de ida y vuelta entre la teoría y realidad empírica. Así, tanto el epígrafe sobre las potencialidades y oportunidades como el que versa sobre el análisis de los retos y contradicciones, introducen discusiones vinculadas a los diferentes intentos de teorizar la Economía Solidaria en sus múltiples vertientes, y también referencias a la todavía relativamente escasa evidencia empírica sobre las organizaciones y procesos de Economía Solidaria.

\section{La Economía Solidaria como generadora de otros modelos de empresa}

\subsection{La práctica organizativa de la Economía Solidaria}

La construcción teórica del concepto de Economía Solidaria está intrínsecamente vinculada a las prácticas de las organizaciones que se reconocen como tales. De hecho, mucha de la todavía escasa literatura sobre la cuestión se ha generado en el contexto de experiencias particulares y en las redes de organizaciones de Economía Solidaria. Este es el caso de la Red de Economía Alternativa y Solidaria del Estado Español (REAS Red de Redes), configurada como una federación de redes territoriales y sectoriales de organizaciones autoidentificadas como de Economía Solidaria en base al compromiso que manifiestan con la denominada Carta de la Economía Solidaria, carta que recoge los principios y valores ${ }^{5}$ de la Economía Solidaria según los entiende la red.

\footnotetext{
${ }^{4}$ El autor alude a su vez a la existencia de un cuerpo de literatura que analiza la experiencia desde una perspectiva crítica, grupo en el que incluye su propio trabajo al respecto.

5 http://www.economiasolidaria.org/carta.php
} 
La carta identifica una serie de valores universales que «deben regir la sociedad y las relaciones entre toda la ciudadania: equidad, justicia, fraternidad económica, solidaridad social y democracia directa» (Carta de la ESOL, 2011).

Se identifican además una serie de principios que deben inspirar la lógica organizativa, la práctica empresarial y el modelo de gestión de la organización, tanto hacia el interior de la misma como en las relaciones con el entorno en que se encuentra inmersa.

En palabras de Askunze (2013), coordinador general de REAS Euskadi, esta carta es

un documento que presenta los seis principios (Equidad, Trabajo, Sostenibilidad Ambiental, Cooperación, Sin Fines Lucrativos; Compromiso con el Entorno) que dotan de cohesión política y orientan las prácticas e iniciativas del movimiento, además de hacer del espacio de la Economía Solidaria un sector específico y delimitado, siempre en relación con otros movimientos de las diversas economías críticas.

Es interesante apuntar cómo el texto de la carta ${ }^{6}$, además de las referencias a los y principios clásicos del Cooperativismo y la Economía Social mencionados anteriormente, plantea vínculos directos con la perspectiva de la llamada Economía Crítica ${ }^{7}$ (Martínez y Álvarez, 2008), particularmente en lo relativo a la introducción de aspectos relacionados con la Economía Feminista, la Economía Ecológica y la Autogestión. Así por ejemplo, tanto el nombre del segundo principio (Trabajo), como su desarrollo desde una perspectiva amplia que visibilice los trabajos reproductivos y los trabajos de cuidados, pueden entenderse desde la Economía Feminista. Igualmente, la denominación del cuarto principio (Sostenibilidad Ambiental), junto a las referencias al decrecimiento o la reducción de la huella ecológica constituyen un posicionamiento en la dirección de la lógica de la Economía Ecológica.

Encontramos además que en la carta se hace una referencia explícita a la autogestión. Esto introduce un matiz diferencial respecto a las apelaciones genéricas a la participación de los trabajadores, el desarrollo personal y la toma de respon-

${ }^{6}$ Conviene señalar que hay dos versiones de la Carta de la Principios de la Economía Solidaria. La más reciente data del 2011 y se observan con respecto a la anterior (datada en el ańos 2000) algunas diferencias que hablan de la evolución del concepto de Economías Solidaria en el contexto de la red. Para una discusión detallada sobre este asunto y sobre la Economía Solidaria en el Estado Español, ver Pérez de Mendiguren (2014), capítulo 4.

7 Como indican Martínez y Álvarez (2008), el término Economía Crítica ha sido acuñado en el contexto del Estado Español para hacer referencia al pensamiento conjunto de un grupo de economistas y científicos sociales, que reconocen un profundo desacuerdo con el paradigma ortodoxo de la economía. Este grupo se mueve en torno a la Revista de Economía Crítica (http:// revistaeconomiacritica.org/) y a las Jornadas de Economía Crítica organizadas desde 1987 en diversas ciudades del Estado (http://www.economiacritica.org/). 
sabilidades, que remite a los debates sobre los significados de la participación y la democracia organizativa en el contexto de la construcción de proyectos sociales alternativos como los que se proponen desde la Economía Crítica.

La plasmación práctica de estos principios puede observarse en la forma y los procesos que adoptan muchas de las experiencias vinculadas con la Economía Solidaria, algunos de ellas en sectores fuertemente condicionados por la lógica de empresas multinacionales, como es el financiero. Así por ejemplo, en el Estado Español, las experiencias de FIARE Banca Ética y Coop57 y sus mo$\operatorname{delos}^{8}$ de negocio bancario y parabancario, ofrecen una interesante ejemplo de cómo se puede construir y gestionar entidades financieras desde procesos colectivos autogestionarios, con base en la agregación de redes de ciudadanía comprometida individual y colectivamente con la transformación social. Ambas experiencias despliegan una lógica organizativa alternativa a la que opera en las empresas financieras de carácter capitalista, y ponen las finanzas al servicio de la construcción de la Economía Solidaria y, en último término, de procesos que tienen como objetivo central la sostenibilidad de la vida (Gassiot 2013; De la Cruz y Sasia 2012; Sasia y De la Cruz, 2008).

De igual manera, en el ámbito de la producción y distribución de alimentos, están surgiendo en múltiples lugares del mundo, redes de cercanía que ponen en contacto productores y consumidores, y que desde una lógica de soberanía alimentaria con carácter agroecológico, orquestan modelos organizativos con diferentes grados de formalización, que pueden abarcar desde la gestión conjunta de las decisiones de producción, hasta la cadena de comercialización y distribución a escala local o regional.

Esta misma lógica de generación de circuitos y cadenas de valor de y para la Economía Solidaria, está en la base de los procesos de construcción de mercados sociales que se están poniendo en marcha en diferentes lugares del Estado Español ${ }^{9}$.

También en el ámbito de la producción de bienes y servicios existen una gran variedad de organizaciones de Economía Solidaria que están experimentando con modelos de gestión que buscan posibilitar el sostenimiento financiero de la organización, sin que esto suponga la renuncia la consecución de los objetivos sociales y/o medioambientales que están en la base de su surgimiento. Así por ejemplo, en el caso de las llamadas Empresas de Inserción Laboral ${ }^{10}$, su de-

8 www.fiarebancaetica.coop; www.coop57.coop

9 http://mercadosocial.konsumoresponsable.coop/

10 Las Empresas de Inserción (EI) espańolas están sujetas a la Ley Estatal de Empresas de Inserción 44/200, de 13 de diciembre. Complementariamente, las diferentes comunidades autónomas, en el desarrollo de sus competencias, han ido desarrollado regulación específica para este tipo de empresa en su territorio. En el caso de la Comunidad Autónoma del País Vasco, el Decreto 182/2008, de 11 de noviembre, modificando el anterior decreto que databa del año 2000, regula la calificación de EI y establece el procedimiento de acceso a las mismas y su registro. 
sarrollo ha sido ampliamente estudiado (Gil y Ramos, 2013; Marcuello y Marcuello, 2010; Veciana, 2007). La casuística es amplia, pero por mencionar un ejemplo estudiado por uno de los autores de este trabajo, en el caso de los Traperos de Emaús en el Estado Español, Pérez de Mendiguren y Villalba (2013) analizan como la flexibilidad para adaptarse al entorno cambiante, la capacidad para movilizar múltiples recursos (mercantiles, no mercantiles, monetarios y no monetarios), y las diferentes visiones sobre como permanecer fieles al ethos de la organización, han dado lugar a cuatro modelos diferentes de organización de Traperos, con lógicas sociales similares pero con muy diferentes lógicas organizativas en cuanto a sus formas jurídicas, sus modelos de liderazgo y participación, o sus lógicas de crecimiento organizativo.

Desde una perspectiva internacional, las organizaciones y las lógicas de la Economía Solidaria están experimentando un importante auge. Así por ejemplo, Brasil, es quizá uno de los lugares del mundo donde más se ha consolidado en la última década, tanto en el desarrollo de experiencias y redes de organizaciones, como en su institucionalización en forma de políticas públicas que la apoyan. El segundo proceso de mapeo de la Economía Solidaria brasileña, finalizado en el 2013, sacó a la luz la existencia de cerca de 20.000 emprendimientos de este tipo (Gaiger 2008, 2007). De la misma manera, en el caso argentino, las experiencias de empresas recuperadas por sus trabajadores (Ruggeri et al., 2011; Vieta 2010; Ruggeri 2010), están aportando ejemplos muy interesantes de experiencias de autogestión en contextos muy hostiles a la consolidación de estas organizaciones, y en ausencia de reconocimiento legal o apoyo institucional para las mismas ${ }^{11}$.

\subsection{Algunos aspectos teóricos que fundamentan la lógica de estas organizaciones}

Desde un punto de vista teórico, el potencial trasformador de la Economía Solidaria se fundamente desde diferentes perspectivas teóricas y con aportaciones originadas en distintos lugares del planeta, y que por lo tanto reflejan la idiosincrasia propia de las organizaciones de referencia en cada región y contexto socioeconómico. Particularmente relevantes, son algunas de las aportaciones que llegan desde Europa y América Latina en la medida en que éstas han influenciado el trabajo académico y empírico en otras regiones y países del mundo. Está fuera de las posibilidades de este texto presentar cada una de ellas en detalle, aunque un análisis más pormenorizado de las mismas puede encontrarse en Pérez de Mendiguren (2014); Laville y García (2009) o Hintze (2010) entre otros. Sin embargo, presentamos a continuación un breve repaso por algunos de los conceptos teóricos que entendemos como más relevantes.

11 Según se escriben estas líneas, llegan noticias de que una de las empresas más paradigmáticas de este movimiento en la Ciudad de Buenos Aires, el hotel Bauen, ha sido definitivamente entregada a los trabajadores que la gestionan desde el 2003 (Machado, 2015). 
Desde una perspectiva europea, García (2010) identifica una serie de "potencias o capacidades trasformadoras" en el sector de la Economía Solidaria, que resume en cinco. La primera emana de entender que las prácticas del sector encierran una suerte de "embrión de la futura economía postcapitalista, en el sentido de que prefiguran cómo podrán ser la mayoría de las empresas de una sociedad ecosocialista» (ibid.:33).

Esto se debe a sus prácticas democráticas, su mínimo nivel de desigualdad entre los miembros, su mayor sostenibilidad ecológica con respecto a las capitalistas, y su capacidad para ser al menos tan eficientes como las anteriores en la producción de bienes y servicios.

La segunda se refiere a que estas organizaciones crean ciudadanía activa y comunidad entorno a prácticas de producción, consumo e inversión de naturaleza no capitalista. En este mismo sentido, la tercera se refiere a la posibilidad que ofrecen para conformar nuevos sujetos de cambio social vinculados a la producción directa de valores contrahegemónicos. La cuarta plantea estas organizaciones como «laboratorios de ensayo» en los que se trata de resolver cuestiones de gestión participativa, de los modelos de encaje de los incentivos individuales en procesos colectivos, o las cuestiones relacionadas con la reconversión ecológica de las empresas. En esta misma línea, y aunque García (2010) no lo menciona explícitamente, podría incluirse las experiencias que, desde la Economía Feminista se preguntan por las posibilidad de construcción de organizaciones empresariales no heteropatrialcales ${ }^{12}$.

Finalmente, y en la medida en que todo lo anterior opera, el quinto y sexto grupo de razones se refieren al efecto «escaparate» y la fuente de inspiración que estas organizaciones ofrecen a todas aquellas personas y colectivos que investigan sobre formas y alternativas para la transformación y/o superación del capitalismo.

En este misma línea, y ahondando en las claves organizativas que permiten articular los anteriormente citados potenciales, algunos autores y autoras de referencia en el marco de la Economía Solidaria europea identifican los conceptos de hibridación y co-construcción de la oferta y la demanda como elementos centrales para estas organizaciones (Laville, 2009, 2004; Defourny y Nyssens, 2006; Laville y Nyssens, 2000). En cuanto a la primera, la capacidad para hibridar ${ }^{13}$ puede ser entendida como una capacidad estratégica para movilizar y acceder a recursos y capacidades provenientes tanto de la economía mercantil, como de la no mercantil (en gran medida vinculados a la lógica de redistribución propia del

12 En esta línea de pensamiento ver Jubeto et al. (2014); Pérez-Orozco (2014).

13 Para un análisis de las implicaciones de las estrategia de la hibridación de las Empresas de Inserción Laboral en el contexto europeo, y los factores que influyen en la sostenibilidad de las mismas ver Bode, Evers and Schulz (2006). 
sector público) y la no monetaria (fundamentalmente derivados de procesos de reciprocidad). Esto permitiría evitar dependencias excesivas de recursos mercantiles o de la financiación pública, y consolidar la actividad de la organización al generar mayor autonomía y viabilidad financiera.

La idea de la co-construcción ${ }^{14}$, por su parte, se refiere al establecimiento de procesos de decisión que asuman la coexistencia de trabajo remunerado y voluntario, y la generación de espacios de carácter público que abran las organizaciones y posibiliten el encuentro de demandas provenientes de diferentes tipos de agentes, con grados de implicación variables. Entre estos agentes se encuentran: usuarios, voluntarios, representantes de otras organizaciones, familiares, profesionales, autoridades públicas, etc.

Por otro lado, y volviendo la mirada hacia América Latina, cabe destacar las aportaciones teóricas derivada de la experiencia brasileńa anteriormente citada, y que entre otras, se refiere a lo que autores como Luz Inazio Gaiger consideran como la existencia constatable de una serie de ventajas con las que cuentan los emprendimientos autogestionados. En su opinión, estas ventajas se sostienen en: la condición dual de copropietario y gestor de las personas en estos emprendimientos; en su capacidad para la toma de decisiones; y en la recuperación de la autoestima como trabajador/a asociada a mayores condiciones de dignidad y significatividad en el trabajo (Gaiger y Da Silva, 2011).

En palabras del propio autor:

el éxito de estas iniciativas depende de su capacidad de articular una lógica emprendedora - de búsqueda de resultados por medio de la acción planificadas y de la optimización de factores productivos, humanos y materiales - y una lógica solidaria, de manera que la cooperación funcione como la fuente de la racionalización económica, produciendo efectos tangibles y ventajas reales, comparativamente con la acción individual y la cooperación no solidaria (...). Cuando estas palancas funcionan, permiten que tales iniciativas sean superiores al trabajo artesanal e individualizado de los pequeńos productores, más productivas que la empresas convencionales correspondientes y más recompensadoras que aquella basadas en el trabajo asalariado (Gaiger y Dos-Anjos, 2011: 260-261, énfasis ańadido).

Como podemos observar en la tabla 1, las razones aportadas por el autor pueden clasificarse en base a criterios que se fundamentan en los enfoques contemporáneos de gestión estratégica de organizaciones empresariales (Guerras y Navas, 2007; Grant, 1996). Se pretende así ilustrar la idea implícita en Gaiger sobre el potencial estratégico de los recursos intrínsecos a los emprendimientos

14 Laville y Nyssens (2000) se basan en la evidencia empírica aportada por más de 69 estudios de caso sobre experiencias en Francia y Bélgica para ilustrar este proceso en el caso de los llamados servicios de proximidad. 
de Economía Solidaria. Esta potencialidad puede traducirse en ventajas tanto por el lado de los costes, como por las posibilidades de diferenciación.

Tabla 1

Ventajas de los emprendimientos asociativos autogestionados

\begin{tabular}{|c|c|c|}
\hline Tipo ventaja & Elementos constitutivos & Razones \\
\hline \multirow{3}{*}{$\begin{array}{l}\text { Reducción de } \\
\text { costes }\end{array}$} & $\begin{array}{l}\text { Reducción costes internos } \\
\text { de transacción }\end{array}$ & $\begin{array}{l}\text { - Menores costes supervisión y control. } \\
\text { — Reducción de costes de información y } \\
\text { motivación. }\end{array}$ \\
\hline & Flexibilidad de ajuste & $\begin{array}{l}\text { - Garantía de empleo. No ajustes vía des- } \\
\text { pido. } \\
\text { — Asunción de reducciones salariales, } \\
\text { cambios en jornada de trabajo y otros } \\
\text { ajustes de capacidad y demanda. }\end{array}$ \\
\hline & $\begin{array}{l}\text { Reducción costes de ca- } \\
\text { lidad }\end{array}$ & $\begin{array}{l}\text { - Actitud colaboradora. } \\
\text { - Menor rotación fuerza de trabajo. } \\
\text { - Relación desempeño organizativo y be- } \\
\text { neficio personal. } \\
\text { - Menor absentismo, desperdicio y tiem- } \\
\text { pos ociosos. }\end{array}$ \\
\hline \multirow[b]{2}{*}{$\begin{array}{l}\text { Diferenciación } \\
\text { creativa }\end{array}$} & $\begin{array}{l}\text { Alineamiento con objeti- } \\
\text { vos y valores }\end{array}$ & $\begin{array}{l}\text { - Compromiso, innovación y creativi- } \\
\text { dad. }\end{array}$ \\
\hline & $\begin{array}{l}\text { Genética de cooperación } \\
\text { y participación como ven- } \\
\text { taja en la organización del } \\
\text { trabajo }\end{array}$ & $\begin{array}{l}\text { - Alineamiento con métodos modernos } \\
\text { de gestión que se basan en la confianza, } \\
\text { participación y cooperación. } \\
\text { - Células trabajo, grupos multifunción, } \\
\text { enriquecimiento de puestos de trabajo, } \\
\text { gestión calidad. }\end{array}$ \\
\hline
\end{tabular}

Fuente: Pérez de Mendiguren (2014), basado en Gaiger 2007.

Siguiendo con las aportaciones teóricas que abordan los elementos diferenciales sobre los que se construyen las lógicas organizativas de los emprendimientos de Economía Solidaria, es necesario mencionar el trabajo del autor chileno Luis Razeto, y su conceptualización del llamado «Factor C», que denota la forma en que la solidaridad propia de estos emprendimientos se materializa en cooperación, colaboración, comunicación, comunidad, compartir, colectividad..., conceptos nombrados con palabras que empiezan por la letra C. Razeto propone cuatro hipótesis relacionadas con el funcionamiento de este factor en las organizaciones solidarias. 
La primera hipótesis se refiere a que este factor se constituye en fuente de eficiencia ${ }^{15}$ para las organizaciones que articulen su funcionamiento en torno al mismo, es decir, que lo tengan como categoría organizadora:

El Factor $C$ significa que la formación de un grupo, asociación o comunidad que opera cooperativa y coordinadamente, proporciona un conjunto de beneficios a cada integrante, y un mejor rendimiento y eficiencia a la unidad económica como un todo, debido a una serie de economía de escala, economías de asociación y externalidades positivas implicadas en la acción común y comunitaria (Razeto, 2005: 8).

La tabla 2 resume las principales razones que ofrece Razeto a la hora de justificar esta hipótesis.

Tabla 2

El Factor $C$ y la eficiencia organizativa. Causas y efectos

Efectos económicos de la solidaridad

1. Cooperación en el trabajo

2. Uso compartido conocimientos

3. Adopción colectiva de decisiones

4. Más equitativa distribución de B. ${ }^{\circ}$

5. Clima laboral favorable

6. Reducción conflictividad laboral

7. Satisfacción necesidades relacionales

Mejoras en eficiencia económica y social

Mejor rendimiento individual y colectivo $\rightarrow$ Mayor eficiencia.

Ahorro en costos (economía de información).

Mayor motivación y compromiso por tomar parte en la planificación.

Motivación para el esfuerzo común.

Personas mejor socializadas e integradas en el proyecto.

Menos intereses antagónico y más cauces de resolución conflictos.

Beneficios derivados de buena convivencia en el proceso de trabajo.

8. Desarrollo integral

Capacidad de articular positivamente vida personal y comunitaria.

9. Impacto positivo sobre comunidad Transformación hacia una sociedad más justa, libre y solidaria.

Fuente: Pérez de Mendiguren (2014), basado en Razeto (2005, 1997).

15 Razeto pone gran énfasis en la necesidad de recuperar para la ESOL el lenguaje de la economía y los conceptos como eficiencia, utilidades, excedentes, producción, mercados... Desde su punto de vista, "muchas de las debilidades del cooperativismo y de otros modos no-capitalistas se originan en esta dificultad para asumir conceptos económicos necesarios" (Intervencion de Razeto en Guerra, 2007). 
La segunda hipótesis deriva de la anterior, y establece que el plus de eficiencia y productividad es de tal magnitud que compensa las múltiples deficiencias en la dotación del resto de factores productivos que se observa en las experiencias de Economía Solidaria. Estas deficiencias de orden cuantitativo y cualitativo son, según Razeto, claramente suplidas por la disponibilidad del Factor $C$, que se erige así en el factor oculto y no remunerado que permite explicar el éxito de estos emprendimientos en contexto tan adversos a su desarrollo.

La tercera hipótesis plantea que, al igual que con el resto de factores, existe Factor $C$ de alta y baja calidad. Razeto explica que el Factor $C$ de baja calidad está asociado a las empresas de capital, que lo utilizan de forma instrumental para asegurarse el grado de coherencia interna necesario para cumplir sus objetivos maximizadores. Desde su punto de vista esto también ocurre con ciertas organizaciones clásicas de la Economía Social, que han ido perdiendo calidad de este factor en la medida en que ha sido subordinado a la lógica de algún otro. Desde esta perspectiva, los emprendimientos solidarios serían empresas intensivas en Factor C. Además, el Factor $C$, presenta una "alta sustituibilidad recíproca» (Razeto, 2005) con el resto de los factores, lo que en palabras de Razeto significa que los puede sustituir con facilidad y en elevada proporción, pudiendo generar además ahorros relativos en costos derivados de esta sustitución (lo que empuja a aumentar la intensidad relativa de esta factor en las empresas de Economía Solidaria).

Finalmente, la última hipótesis se refiere a la acumulación del Factor $C$, cuestión sobre la que pueden distinguirse un ámbito, interno y otro externo. En cuanto a su acumulación hacia el interior de los emprendimientos, Razeto entiende que si bien la viabilidad de los mismos depende de su capacidad para reproducir y mejorar todos sus factores, es crucial que la acumulación se produzca en Factor $C$ (mejorando y extendiendo su calidad) y en factor trabajo asociativo (de calidad y con ganas de contribuir colectivamente). Cuando esto no ocurre así, aparece el peligro de perder la lógica solidaria del emprendimiento.

En los referente a la acumulación de Factor $C$ en el orden externo, ésta se refiere a que la lógica de la solidaridad puede y debe extenderse al ámbito de la relaciones entre organizaciones y territorios, apoyándose en la creación de redes y circuitos económicos solidarios de producción, consumo, distribución y financiación (Lopera y Mora, 2009). Se constituiría así un sector autónomo con racionalidad propia que irá alcanzando crecientes niveles de autonomía con respecto a las organizaciones capitalistas. En esta misma línea de reflexión, el filósofo brasileño Euclides Mance sostiene que la estrategia para que la Economía Solidaria se construya como una alternativa global de carácter post-capitalista pasa por el establecimiento y consolidación de "Redes de Colaboración Solidaria» (Mance, 2008). 


\section{Retos teóricos y prácticos de las experiencias de Economía Solidaria}

Como hemos indicado en la introducción, vamos a concluir el artículo con una reflexión crítica sobre las paradojas y tensiones que soportan los modelos organizativos vinculados a la Economía Solidaria, tanto desde una perspectiva teórica como empírica.

Comenzando con las cuestiones de orden teórico, Ould (2014) plantea una serie de interesantes reflexiones con respecto al propio concepto de solidaridad y el significado que se le asigna en los principales marcos teóricos sobre la Economía Solidaria. Desde su punto de vista, algunos de los planteamientos que se hacen en este sentido están basados en una suerte de moralización de los agentes económicos, que por sí sola no aseguraría que se establecieran nuevos patrones de comportamiento social conducentes a transformaciones significativas. La apelación a la lógica de la reciprocidad que hace la Economía Solidaria no sería suficiente en este sentido. La autora apunta a la necesidad de un análisis mucho más preciso del proyecto político que se encierra bajo la idea de democratización de la economía y su gestión, tanto en el ámbito de las organizaciones como en la construcción de políticas pública y las dinámicas sociales.

En este sentido, la apelación a la hibridación y la co-construcción como elementos estratégicos de los modelos de negocio de empresas de Economía Solidaria no pueden convertirse en una apelación de orden neutral y ausente de conflicto. En la medida en que afecten a la construcción de la identidad de las organizaciones y sus personas, y a la legitimidad interna y externa de las mismas, estas estrategias deben ser abordadas desde la perspectiva del conflicto y de los mecanismos de gestión del mismo. En este sentido, los modelos de gobernanza democrática de corte autogestionario a los que se aspira necesitan mucho más trabajo teórico y empírico en el sector. La apelación genérica a que la actividad de personas y organizaciones en la Economía Solidaria está organizada en base a principios normativos, a la solidaridad, a la autogestión, o a la existencia de factores integradores propios de las organizaciones de Economía Solidaria como el mencionado Factor $C$, no es garantía suficiente de que estas organizaciones sean capaces de conseguir los objetivos de transformación que se plantean.

De hecho, y como señalan Sarasua y Udaondo (2004), la propia lógica de la autogestión está envuelta en múltiples tensiones y paradojas, ya que puede asociarse a experiencias con importantes diferencias en cuanto a su potencial transformador. Incluso cabe su utilización en el marco de las tendencias del management moderno para incentivar la implicación de los trabajadores en aras de la mayor eficiencia y productividad dentro de una lógica capitalista. Existe así el riesgo de que la autogestión se deslice hacia un economicismo productivista, convirtiéndose en una mera práctica de organización interna, y generando un «enfriamiento democrático» que diluya la participación real de las personas en 
el contexto de una complejidad organizativa que las aleja del poder real para la toma de decisiones.

Por su parte, Diefencbac y Sillince (2011) plantean que en organizaciones y redes organizativas basadas en la cooperación, y que tratan de establecer modelos organizativos libres de jerarquía formal, se manifiesta una tendencia a la aparición de estructuras jerárquicas informales que se consolidan a través de los procesos, contenidos y prácticas comunicativas de la organización o la red. Este proceso, indican además, no estaría inducido por la existencia de prácticas no-éticas de carácter individual o colectivo, sino por el resultado de diferencias en los procesos comunicativos. La jerarquía informal que se genera se trasladaría además a los procesos de toma de decisiones y de asignación de recursos. Por lo tanto, se acabarían reproduciendo estructuras formales de poder y control al interior de la organización y la red, minando así sus propios objetivos fundacionales. Utilizando la lógica de Razeto, podríamos denominar a este proceso como de destrucción de Factor $C$ (frente a la acumulación de la que el autor habla).

Por otra parte, y si atendemos a la evidencia empírica generada en el campo de la Economía Social, el riesgo de que se abra una brecha entre los principios y la prácticas es un tema recurrente en la literatura sobre modelos de gobernanza ${ }^{16}$ en cooperativas (Heras-Saizarbitoria, 2014; Shaw y Carter, 2007). La literatura apunta a que el crecimiento en tamaño y complejidad organizativa, o la creciente importancia de los valores mercantiles en relación a los sociales, pueda deteriorar la calidad democrática de la organización. Esto podría tener como resultado una tendencia hacia modelos de control gerencial profesionalizado que se distancian cada vez más de los valores cooperativos (Spear 2006; Malo y Vezina, 2004).

Por lo tanto, conviene no perder de vista que las organizaciones de Economía Solidaria no están exentas de caer en los mismos procesos. En cierta medida y utilizando un símil geográfico, se puede afirmar que estas empresas presentan un cierto carácter de organización frontera (Pérez de Mendiguren, 2014: 197). Y es que, si bien son la expresión práctica del intento de construir otra economía mediante otra forma de hacer empresa, también tienen que ser capaces de sobrevivir en el marco de las "reglas del juego", relaciones e instituciones que caracterizan al sistema económico capitalista dominante. La dinámica de la competencia en el mercado y, en general, la necesidad de operar en el marco de instituciones económicas diseñadas desde la óptica del capital y no desde las necesidades de las personas, es fuente de importantes contradicciones y tensiones en estas empresas, que tienen que hacer compatible la consecución de objetivos

16 Gobernanza, del inglés governance, se refiere en el contexto de las organizaciones al proceso de toma de decisiones y al proceso por el que las decisiones son puestas en marcha o no 
sociales y ambientales, a la vez que ser capaces de asegurar los ingresos necesarios para sustentar su actividad.

En muchos casos, estos objetivos no son compatibles en el corto plazo y plantean disyuntivas complejas que pueden acabar minando la legitimidad de la organización, particularmente cuando la consecución de objetivos de corte financiero implica el sacrificio de los compromisos sociales y las intenciones transformadoras de la propia empresa. En otros casos, sus propias condiciones de actividad (trabajadores con necesidades especiales de formación, o con situaciones personales o sociales de exclusión etc.) dificultan la puesta en marcha de mecanismos de gestión típicos de empresas tradicionales que operan en el mismo sector de actividad.

La evidencia empírica disponible, al menos en el Estado Español, apunta claramente en esta dirección. Así, en un estudio que realizamos sobre los tipos de crisis a los que se enfrentan las empresas de Economía Solidaria del Estado Español, Guridi et al. (2011) analizaron el caso de diez entidades de diferentes sectores, y con diferentes recorridos en el seno de la Economía Solidaria. El estudio concluye que los aspectos centrales que caracterizan a los procesos de crisis vividas por las organizaciones, tienen que ver con la dificultad para mantener la identidad, la legitimidad y la coherencia organizativa en situaciones inducidas por factores de orden externo e interno. Entre los externos destacan los que dificultan la adecuación del modelo organizativo a los cambios en el entorno social, institucional, o a las exigencias de la competencia en el mercado. Entre los internos aparecen aquellos asociados a las dinámicas de crecimiento y control de la organización, la gestión de los liderazgos, o las crisis en los modelos de participación. La tabla 3 ofrece un resumen de las principales conclusiones del estudio.

Entre los aspectos reseñables del mismo, la gestión de los procesos de liderazgo, y sus mecanismos de reproducción destacan una vez más como elementos críticos en las organizaciones de Economía Solidaria. Estos liderazgos a su vez están fuertemente relacionados con las características de las personas que crearon o impulsaron la organización, particularmente si éstas permanecen en la organización. En el caso de las empresas del Estado, las personas fundadoras suelen tener un perfil activista con un fuerte compromiso social que tiene origen, bien en comunidades religiosas de base y organizaciones vinculadas a la iglesia católica; bien en la lucha sindical y política, o bien de personas con larga trayectoria en movimientos sociales.

Su permanencia en la organización suele derivar en formas de liderazgo individual, de corte carismático. Este tipo de liderazgos tienen la ventaja de funcionar como poderosos elementos de cohesión organizativa, además de mantener un alto grado de legitimidad interna para tomar decisiones con respecto a asuntos conflictivos, y externa en su relación con otros agentes sociales. Sin embargo, también puede presentar el inconveniente de actuar como freno para los 
Tabla 3

Empresas de ESOL. Tipología de crisis, estrategias y aprendizajes

\begin{tabular}{|c|c|c|}
\hline Tipología de crisis/cambios & Estrategias adoptadas & Aprendizajes adquiridos \\
\hline $\begin{array}{l}\text { Adecuación del modelo de or- } \\
\text { ganización institucional }\end{array}$ & $\begin{array}{l}\text { Cambios en la natura- } \\
\text { leza jurídica de las or- } \\
\text { ganizaciones }\end{array}$ & $\begin{array}{l}\text { Relevancia de la flexibilidad para adaptar las for- } \\
\text { mas jurídicas de las organizaciones a las condicio- } \\
\text { nes en cada momento. }\end{array}$ \\
\hline \multirow{5}{*}{$\begin{array}{l}\text { Gestión y organización in- } \\
\text { terna: } \\
\text { - Crisis de crecimiento } \\
\text { - Crisis de liderazgo } \\
\text { - Crisis de modelo de parti- } \\
\text { cipación } \\
\text { - Crisis de legitimidad }\end{array}$} & \multirow{2}{*}{$\begin{array}{l}\text { Establecer planes de } \\
\text { formación y capacita- } \\
\text { ción }\end{array}$} & $\begin{array}{l}\text { Importancia de contar con un núcleo de personas } \\
\text { que lideren el proyecto a lo largo de la vida de la } \\
\text { institución. }\end{array}$ \\
\hline & & $\begin{array}{l}\text { Subrayar el valor de la convicción en el conjunto } \\
\text { del proyecto e implicación de las personas. }\end{array}$ \\
\hline & $\begin{array}{l}\text { Elaboración de Planes } \\
\text { Estratégicos }\end{array}$ & $\begin{array}{l}\text { Necesidad de reforzar la identidad de la organi- } \\
\text { zación. } \\
\text { Importancia de contar con objetivos claros y } \\
\text { compartidos a medio plazo. } \\
\text { Necesidad de adaptar y ampliar canales de par- } \\
\text { ticipación. }\end{array}$ \\
\hline & $\begin{array}{l}\text { Refundación organiza- } \\
\text { tiva }\end{array}$ & $\begin{array}{l}\text { Alineamiento con principios de la Economía So- } \\
\text { lidaria. }\end{array}$ \\
\hline & $\begin{array}{l}\text { Re-establecimiento de } \\
\text { fuentes internas de le- } \\
\text { gitimidad }\end{array}$ & $\begin{array}{l}\text { Revisar motivación e implicación con el pro- } \\
\text { yecto. } \\
\text { Acomodar el proyecto para reconciliar necesida- } \\
\text { des individuales y colectivas. }\end{array}$ \\
\hline \multirow{5}{*}{$\begin{array}{l}\text { Adaptación a las exigencias del } \\
\text { entorno/demanda/mercado }\end{array}$} & $\begin{array}{l}\text { Innovación en produc- } \\
\text { tos, servicios }\end{array}$ & \multirow{2}{*}{$\begin{array}{l}\text { Necesidad de la innovación permanente. } \\
\text { Importancia de la sostenibilidad económica del } \\
\text { proyecto. } \\
\text { Implantar herramientas de gestión adaptadas a } \\
\text { las nuevas realidades. }\end{array}$} \\
\hline & $\begin{array}{l}\text { Búsqueda de nuevos } \\
\text { mercados }\end{array}$ & \\
\hline & $\begin{array}{l}\text { Vinculación con los ac- } \\
\text { tores y grupos sociales }\end{array}$ & \multirow{2}{*}{$\begin{array}{l}\text { Estrechar los lazos con la sociedad y con el en- } \\
\text { torno. } \\
\text { Aprendizaje de otras experiencias. } \\
\text { Mayor impacto a través de acciones organizadas } \\
\text { en red. }\end{array}$} \\
\hline & $\begin{array}{l}\text { Consolidar la participa- } \\
\text { ción en redes }\end{array}$ & \\
\hline & $\begin{array}{l}\text { Búsqueda de apoyo ins- } \\
\text { titucional }\end{array}$ & $\begin{array}{l}\text { Reforzar los lazos con las administraciones pú- } \\
\text { blicas. }\end{array}$ \\
\hline
\end{tabular}

Fuente: Guridi et al., 2011. 
procesos de aprendizaje colectivo y la asunción de responsabilidades. En este mismo sentido, Pérez de Mendiguren y Villalba (2014) identificaron resultados similares en alguno de los modelos organizativos de Traperos de Emaús generados en su estudio.

Por otra parte, se constata también que a pesar de que el sector está bastante feminizado, los liderazgos están muy masculinizados. A pesar de que la presencia de mujeres en puestos de responsabilidad es mucho mayor que en cualquier otro tipo de empresas, su presencia en puestos de dirección es todavía minoritaria. La puesta en marcha de planes específicos de igualdad de género es un reto que empieza a ser abordado en las empresas de Economía Solidaria.

Un segundo aspecto reseñable tiene que ver con la participación de las personas en la dinámica organizativa. Más allá de los procedimientos formales para ejercer la participación, y que en cierta medida dependen del estatuto legal de la organización, el estudio indica que los niveles de implicación y participación varían mucho entre las personas, y que es mucho mayor en los ámbitos de la planificación del trabajo, que en los procesos de evaluación del resultado, o en el ámbito de las decisiones estratégicas. Esto responde a varias razones.

En algunos casos existe una excesiva concentración de responsabilidades en unas pocas personas, fruto de la escasa utilización de los órganos colectivos, y/o a la presencia de fuertes liderazgos individuales. En otros casos, se debe a que el crecimiento organizativo desajusta los mecanismos de participación y de transmisión de la cultura organizativa. Los mecanismos informales que funcionan en organizaciones pequeñas pueden ser insuficientes en organizaciones de mayor tamaño. Por otra parte, la falta de protocolos de inmersión o planes de acogida para las nuevas personas que se incorporan, hace que en algunos casos se pierda de vista la cultura de la organización, la memoria sobre los orígenes, y/o el sentido y la trayectoria de la misma. Esto puede resultar en que las nuevas personas entienden su periplo en la organización como «un trabajo más». En varios de los casos investigados se manifiesta que la trasmisión de la experiencia histórica y la cultura organizativa se realiza a través de mecanismos informales que conduce a relatos excesivamente fraccionados que impiden el aprendizaje colectivo, y que no comunican de una manera clara y fehaciente los valores organizativos, o que impiden aclarar suficientemente el resultado de conflictos o tránsitos organizacionales anteriores y los elementos que contribuyeron a su superación.

Por lo general, las organizaciones son conscientes de la importancia y la validez de la formación interna como instrumentos para hacer frente a estas situaciones, pero en muchos casos, se carece de planes de formación, y en aquellos casos donde se contemplan, su implementación es muy desigual y poco sistemática, con niveles de participación insuficientes. Solamente el $31 \%$ de las iniciativas investigadas afirmaron tener planes de formación interna, y casi la mitad de ellas tenían que ver con formaciones de carácter técnico-profesional 
vinculados al manejo del negocio y la gestión. Son muy pocas las que contemplan programas formativos en materia de Economía Solidaria, medioambiente, feminismo, etc.

Para finalizar, es necesario mencionar las dificultades derivadas de la adaptación de estas organizaciones a las exigencias del mercado. En un contexto de presupuestos públicos decrecientes y de mayor dificultad para conseguir financiación procedente de la labor redistribuidora del Estado, las organizaciones de Economía Solidaria interactúan en situaciones de mercado con empresas privadas. La competencia es, en muchos casos, en términos de precios y en sectores en los que existen serias dificultades para la diferenciación y pocas barreras de entrada para evitar que las ideas, productos y servicios ofertados sean replicados por los competidores. Concurre, además, el hecho de que tanto las formas de interacción mercantil, como sus lógicas de eficiencia monetaria y eficacia en el corto plazo, no responden al «ADN» de las empresas de Economía Solidaria. Por otro lado, los instrumentos y herramientas de gestión empresarial están particularmente diseñados para la lógica de intervención de empresas de capital.

Por lo tanto, se puede seńalar que la actividad y la viabilidad de los emprendimientos están fuertemente condicionadas por el entorno en el que operan, tanto en aquellos casos en los que tienen que someterse a duras condiciones de competencia, como en otros que dependen de las ofrecidas por las administraciones que demandan sus bienes o sus servicios. En cualquiera de los casos, los emprendimientos necesitan adaptarse, aunque ello genere problemas y distorsiones importantes.

En algunos casos, el acceso a fuentes de financiación que posibiliten el futuro de la organización, se ha hecho a costa de implantar una fuerte cultura de la eficiencia clásica. Esto ha supuesto la introducción en la organización de métodos de gestión que han priorizado: la toma rápida de decisiones frente al consenso y la participación; y el ahorro en costes y la estandarización frente a los entornos de trabajo más humanizados. En estos casos algunas personas entienden que el lenguaje empresarial y su lógica se han impuesto, restando a la experiencia gran parte de su poder motivador y creando conflictos entre las personas del taller y el grupo gestor.

En otros casos, la llegada de fuentes de financiación públicas, ha significado la asunción de responsabilidades en la prestación de servicios que históricamente han pertenecido al sector público. Con el paso del tiempo, la organización ha sido penetrada por una dinámica de prestación de servicios que refleja, en gran medida, la agenda de la institución pública que facilita la mayoría de los fondos, a la vez de un crecimiento desordenado (de la mano de la posibilidad de acceder a más fondos y gestionar otros programas) que ha generado una sensación de alienación en las personas participantes. Esto, además, ha provocado problemas 
de contestación interna de los liderazgos tradicionales (que han apostado fuerte por la anterior dinámica), frente a otras personas que consideran que la organización está perdiendo la capacidad y la autonomía de actuación necesaria. Mientras tanto, y ante esa tesitura, las personas nuevas que se integran en la organización corren el peligro de desvincularse de esta discusión sobre legitimidades para dedicarse unilateralmente a su trabajo, corriendo el riesgo de perder perspectiva y desligarse de la fuerte cohesión interna que ha caracterizado históricamente a la organización.

\section{Conclusiones}

La constatación empírica de otros modelos organizativos vinculados a la Economía Solidaria necesita de un ejercicio de teorización que huyendo de visiones excesivamente románticas o mitificadoras, sistematice de alguna forma la diversidad de prácticas organizativas entorno a una serie de ideas fuerza. La consolidación de estas ideas-fuerza, que constituyen finalmente el «ADN» de las entidades de Economía Solidaria, sirve no sólo a un nivel retórico o moralizador, sino que es un elemento fundamental para afrontar, permítasenos la expresión, la batalla de la legitimidad, tanto teórica como organizacional. Esta batalla de la legitimidad con respecto a otras formas organizacionales más capitalistas, es fundamental si estas prácticas aspiran a ser hegemónicas en ese tránsito hacia esa otra economía.

Sin embargo, esta batalla tiene lugar con unas reglas de juego tremendamente complejas para las entidades de la Economía Solidaria, complejidad que se deriva de su carácter de "organizaciones frontera». Esta ubicación fronteriza les presiona doblemente en la medida en que han de mostrar una consistencia en términos de sostenibilidad económica pero también en términos de coherencia discursiva y organizacional. Es decir, su legitimidad se ve cuestionada en dos direcciones opuestas: deben ser proyectos alternativos que sean económicamente viables y sostenibles, pero este hecho de poco serviría si fuera resultado de procesos progresivos de desacople entre retórica y práctica organizacional.

Este artículo ha tratado de sintetizar los puntos distintivos y los principales retos que distintos autores atribuyen a las experiencias de la Economía Solidaria desde la propia evidencia empírica. Así, para los autores mencionados en este artículo las prácticas organizacionales de la Economía Solidaria se distinguen por distintos motivos: por su capacidad transformadora, por el hecho de hibridar recursos y aunar demanda y oferta en la misma estructura, por aunar asimismo una lógica emprendedora y una lógica solidaria, por ser experiencias intensivas en Factor $C$, o por su capacidad de intercooperar a través de redes de colaboración solidaria, por mencionar algunos. 
Sin embargo, estos elementos distintivos que son al mismo tiempo sus principales fuentes de legitimación organizacional, se desarrollan en un contexto que es extraño y muchas veces antagónico a sus propias lógicas de actuación. El hecho de actuar en mercados capitalistas y el tener que adaptarse a este entorno para ser proyectos sostenibles, puede generar una serie de presiones internas muy fuertes en estas entidades. Presiones que tienen que ver con procesos de degeneración de las prácticas autogestionarias y de las dinámicas participativas, procesos que generan un "enfriamiento democrático», o que generan liderazgos que agravan estos procesos degenerativos. La dependencia financiera que condiciona la actividad de estas organizaciones genera procesos de isomorfismo de mercado y de estado, en la medida en que éstas pueden empezar a replicar o a someterse en exceso a lógicas de eficiencia económica capitalista o lógicas de burocratización.

Estos procesos de desacople entre retórica y práctica ya han sido ampliamente documentadas para otro tipo de experiencias más vinculadas a la Economía Social y de ellas podríamos extraer algunos aprendizajes interesantes para las entidades de la Economía Solidaria.

La teoría institucionalista (Meyer y Rowan, 1977) concluye que estos procesos de desacople no siempre ocurren por un distanciamiento de la estructura gerencial o por una apatía de las base social con respecto a las ideas fundacionales, sino que muchas veces ocurren por ser funcionales para el conjunto de la organización. Así, la retórica alternativa serviría para ganar legitimidad externa, mientras que el desacople dotaría a las organizaciones de cierta flexibilidad interna para acometer problemas de carácter más práctico. Estos procesos de desacople también deben ser entendidos en un contexto social muy determinado, contexto marcado por tendencias individualizadoras y destructoras de lazos sociales que hacen que las prácticas solidarias de carácter autogestionario se desarrollen en condiciones adversas.

Por lo tanto, la capacidad para gestionar las contradicciones que se generan por la necesidad de aunar objetivos de corte social con objetivos financieros y dinámicas de corte mercantil es clave en las organizaciones de Economía Solidaria. Conseguir mantener la coherencia interna, la participación y la legitimidad en un contexto de competencia en el mercado y de dificultad de acceso a fondos públicos es un reto complejo. Las experiencias investigadas apuntan a que una cultura de la experimentación, sensata pero sin complejos por el miedo a cometer errores, la capacidad de reforzar lazos internos a través de los liderazgos grupales, la transparencia en la toma de decisiones y la revisión abierta y continua de las estrategias propuestas a la luz de los principios organizativos suponen el camino más sensato. Además de esto, la generación de una red densa de vínculos y capacidad de trabajo con otras organizaciones, y la vinculación al territorio y al entorno social de la experiencia, son elementos fundamentales para hacer frente a las situaciones de crisis. 


\section{Bibliografía}

ASKUNZE, Carlos (2013): «Más allá del capitalismo: alternativas desde la Economía Solidaria", Documentación social, 168, 91-116.

ASKUNZE, Carlos (2007): «Economía Solidaria», en CELORIO, Gema y LÓPEZ DE MUNIAIN, Alicia (coords.), Diccionario de Educación para el Desarrollo, Bilbao, Hegoa, 107-113.

ARRUDA, Marcos (2005): Humanizar lo infrahumano: la formación del ser humano integral: homo evolutivo, praxis y economía solidaria, Barcelona, Icaria Editorial.

ARRUDA, Marcos (2004): «¿Qué es la Economía Solidaria? El renacimiento de una sociedad humana matrística», Ecología Política, 27: 71-76.

BODE, Ingo, EVERS, Adalbert, y SCHULZ, Andreas (2006): "Work integration social enterprises in Europe: can hybridization be sustainable?», en NYSSENS, Marthe (ed.), Social enterprise: At the crossroads of market, public policies and civil society, Londres, Routledge, 237-259.

CHAVES, Rafael, MONZÓN, José Luis y SAJARDO, Antonia (2003): Elementos de economía social, Valencia, Universidad de Valencia.

CORAGGIO, José Luis (2012): Las tres corrientes vigentes de pensamiento y acción dentro del campo de la Economía Social y Solidaria (ESS). Sus diferentes alcances. En: http:// www.socioeco.org/bdf/es/corpus_document/fiche-document-2124.html

CORAGGIO, José Luis (2011): Economía social y solidaria. El trabajo antes que el capital, Quito, Abya Yala.

CORAGGIO, José Luis (ed.) (2009): Qué es lo económico? Materiales para un debate necesario contra el fatalismo, Buenos Aires, Ciccus.

CORAGGIO, José Luis (2007): «Una perspectiva alternativa para la economía social: de la economía popular a la economía del trabajo», en CORAGGIO, José Luis (ed.), La Economía Social desde la periferia. Contribuciones latinoamericanas, Buenos Aires, Altamira, 165-194.

DA ROS, Giuseppina S. (2007): «Economía Solidaria: aspectos teóricos y experiencias», Unircoop, 5(1): 9.

DE LA CRUZ, Cristina y SASIA, Pedro M. (2012): «La banca ética como herramienta de acción política», Revista Icade. Publicación de las Facultades de Derecho y Ciencias Económicas y Empresariales, 80, 185-210.

DEFOURNY, Jacques. y NYSSENS, Marthe (2006): «Defining Social Enterprise», en NYSSENS, Marthe (ed.), Social Enterprise, at the Crossroads of Market, Public Policies and the Civil Society, London \& New York, Routledge, 3-26.

DIEFENBACH, Thomas y SILLINCE, John A. (2011): «Formal and informal hierarchy in different types of organization", Organization Studies, 32(11), 1515-1537.

ETXEZARRETA, Enekoitz y MORANDEIRA, Jon (2012): "Consideraciones conceptuales sobre la Economía Social a la luz de la Ley 5/2011», Revista vasca de economía social. Gizarte ekonomiaren euskal aldizkaria, 8: 7-36.

GAIGER, Luiz I. y DA SILVA, Andressa (2011): «O diferencial do empreendedorismo solidário", Ciências Sociais Unisinos, 47(1), 34-43.

GAIGER, Luiz I. y DOS-ANJOS, Eliene (2012): «Economía Solidaria en Brasil: la actualidad de las cooperativas para la emancipación histórica de los trabajadores», en PINEIRO, Camila (ed.), Cooperativas y Socialismo. Una mirada desde Cuba, La Habana, Editorial Caminos, 245-271 . 
GAIGER, Luiz I. (2008): «A dimensão empreendedora da economia solidária: notas para um debate necessário", Otra Economía, 2(3), 58-72.

GAIGER, Luiz I. (2007): "La economía solidaria y el capitalismo en la perspectiva de las transiciones históricas», en CORAGGIO, Jose Luis, La Economía Social desde la periferia. Contribuciones latinoamericanas, Buenos Aires, Altamira, 79-109.

GARCÍA, Jordi (2010): «La Economía Solidaria: sustento y esperanza», Revista de Economía Solidaria, 2, 25-57.

GASSIOT, Raimon (2013): "Las finanzas éticas como instrumento al servicio de la economía solidaria. La experiencia de Coop57», Cuadernos de Trabajo Social, 26(1), 75-84.

GIL, María Elena y RAMOS, Nieves (2013): «Las empresas de inserción en la economía social y solidaria: perspectivas y propuestas desde FAEDEI», Cuadernos de Trabajo Social, 26(1), 43-53.

GUERRA, Pablo (2013): «Las legislaciones sobre economía social y solidaria en América Latina entre la autogestión y la visión sectorial», Revista de la Facultad de Derecho, 33,73-94.

GUERRA, Pablo (2011): «;Cómo denominar a las experiencias económicas solidarias basadas en el trabajo? Diálogo entre académicos latinoamericanos acerca de la polémica conceptual», Otra Economia, 1(1), 21-27.

GUERRA, Pablo (2010): «La Economía Solidaria en Latinoamérica», Papeles de relaciones ecosociales y cambio global, 110, 67-76.

GUERRA, Pablo (2007): «¿Cómo denominar a las experiencias económicas solidarias basadas en el trabajo? Diálogo entre académicos latinoamericanos acerca de la polémica conceptual», Otra Economía, 1(1), 21-27.

GUERRAS, Luis A. y NAVAS, José E. (2007): La dirección estratégica de la empresa, Madrid, Civitas.

GURIDI, Luis, PÉREZ DE MENDIGUREN, Juan Carlos; IAMETTI, Ana; DEUX, María Victoria; VÁZQUEZ, Gonzalo y URIBE, Amaia (2011): «Experiencias de Economía Social y Solidaria: compartiendo estrategias y aprendizajes», Papeles de Economía Solidaria, 2, 1-77.

GRANT, Robert M. (1996): "Towards a Knowledge-Based Theory of the firm», Strategic management journal, 17(S2), 109-122.

HINTZE, Susana (2010): La política es un arma cargada de futuro: la Economía Social y Solidaria en Brasil y Venezuela, Buenos Aires, CICUS/CLACSO.

HERAS-SAIZARBITORIA, Ińaki (2014): «The ties that bind? Exploring the basic principles of worker-owned organizations in practice», Organization, 21(5), 645-665.

JUBETO, Yolanda; GURIDI, Luis y FERNÁNDEZ-VILLA, Maite (ed.) (2014): Diálogos sobre Economía Social y Solidaria en Ecuador: encuentros y desencuentros con las propuestas para otra economía, Bilbao, Hegoa.

LAVILLE Jean Louis (2010): "The Solidarity Economy: An International Movement", RCCS Annual Review. A selection from the Portuguese journal Revista Critica de Ciências Sociais, 2, 3-41.

LAVILLE, Jean Louis (2009): «Definiciones e instituciones de la economía» en CORAGGIO, José Luis (ed.), ¿Qué es lo económico? Materiales para un debate necesario contra el fatalismo, Buenos Aires, CICCUS, 47-69.

LAVILLE, Jean Louis (2004): «El marco conceptual de la Economía social y solidaria», en LAVILLE, Jean Louis (ed.), Economía social y solidaria. Una visión europea, Buenos Aires, Fundación OSDE-Universidad Nacional de General Sarmiento-Editorial Altamira, 1-25. 
LAVILLE, Jean Louis y GARCÍA, Jordi (2009): Crisis capitalista y Economía Solidaria, Barcelona, Icaria

LAVILLE, Jean Louis y NYSSENS, Marthe (2000): «Solidarity-Based Third Sector Organizations in the "Proximity Services" Field: A European Francophone Perspective», Voluntas: International Journal of Voluntary and Nonprofit Organizations, 11(1), 6784.

LOPERA, Luz D. y MORA, Sol B. (2009): «Los circuitos económicos solidarios: espacio de relaciones y consensos», Semestre Económico 12 (25), 81-93.

MACHADO, Decio (2015): «El Hotel Bauen, para los trabajadores», Diagonal, 28 de noviembre. En: https://www.diagonalperiodico.net/global/28523-hotel-bauen-definitivamente-para-trabajadores.html

MALO, Marie C., y VÉZINA, Martine (2004): "Governance and Management of Collective User-Based Enterprises: Value-Creation Strategies and Organizational Configurations", Annals of public and cooperative economics, 75(1), 113-137.

MANCE, Euclides. (2008): La Revolución de las Redes. La colaboración solidaria como una alternativa pos-capitalista a la globalización actual, Ciudad de México, Varios co-editores.

MARCUELLO, Carmen y MARCUELLO, Chaime (2010): «Las Empresas de Inserción», en MONZÓN, José Luis (dir.): La Economía Social en España en el año 2008 Ámbito, magnitudes, actividades y tendencias, CIRIEC-España, 417-433.

MARTINEZ, Ángel y ÁLVAREZ, Santiago (2008): «La economía crítica y solidaria: perspectivas teóricas y experiencias para la construcción de una economía alternativa», en La situación del mundo 2008. Innovaciones para una economía sostenible. Informe anual del Worldwatch Institute sobre el Progreso hace una Sociedad Sostenible, Barcelona, CIPEcosocial e Icaria, 371-486.

MEYER, John and ROWAN, Brian (1977): «Institutional Organizations: Formal Structure as Myth and Ceremony», American Journal of Sociology 83, 340-63.

MONZÓN, José Luis (2003): «El cooperativismo en la historia de la literatura económica», CIRIEC-ESPAÑA, Revista de economía pública, social y cooperativa, 44, 9-32.

MONZÓN, José Luis y CHAVES, Rafael (2012): La Economía Social en la Unión Europea, Bruselas, Comite Economico y Social Europeo.

MONZÓN, José Luis y CHAVES, Rafael (2011): «Social Economy, an international perspective. Introduction", CIRIEC-España, revista de economía pública, social y cooperativa, 73, 5-8.

MOULAERT, Frank y AILENEI, Oana (2005): «Social economy, third sector and solidarity relations: A conceptual synthesis from history to present», Urban Studies, 42(11), 2037-2053.

OULD, Pepita (2014): «What does 'solidarity economy'mean? Contours and feasibility of a theoretical and political project», Business Ethics: A European Review, 24(4), 425-435.

PÉREZ DE MENDIGUREN, Juan Carlos (2014): Debates conceptuales y aspectos organizativos de la Economía Solidaria: el caso de REAS Euskadi. Bilbao, Tesis Doctoral. Universidad del Pais Vasco UPV/EHU.

PÉREZ DE MENDIGUREN, Juan Carlos (2013): «Social enterprise in the development agenda. Opening a new road map or just a new vehicle to travel the same route?», Social Enterprise Journal, 9(3), 247-268.

PÉREZ DE MENDIGUREN, Juan Carlos y ETXEZARRETA, Enekoitz (2015a): «Economía Social y Solidaria: Desde la negación al reconocimiento mutuo», Revista de Economía Mundial, 40,123-144. 
PÉREZ DE MENDIGUREN, Juan Carlos y ETXEZARRETA, Enekoitz (2015b): «Los debates en torno a la Economía Social y Solidaria», Boletín de Recursos de Información 4, 1-12.

PÉREZ DE MENDIGUREN, Juan Carlos, ETXEZARRETA, Enekoitz y GURIDI, Luis (2009): «Economía Social, Empresa Social y Economía Solidaria: diferentes conceptos para un mismo debate», Papeles de Economía Solidaria, 1, 1-41.

PÉREZ DE MENDIGUREN, Juan Carlos y VILLALBA, Unai (2013): «Los modelos de Traperos de Emaús en el Estado y su respuesta a la crisis», Revista de Estudios Cooperativos-REVESCO, 113, 150-179.

PÉREZ-OROZCO, Amaia (2014): Subversión feminista de la economía. Aportes para un debate sobre el conflicto capital-vida, Madrid, Traficantes de Sueños.

RAZETO, Luis (2005): «El concepto Solidaridad», Pensamiento Critico Latinoamericano. Conceptos Fundamentales, 3, 971-985.

RAZETO, Luis (1997): Factor C, Charla en la Escuela Cooperativa «Rosario Arjona» CECOSESOLA. En: http://www.economiasolidaria.org/files/el_factor_c.pdf

RUGGERI, Andrés (2010): Las empresas recuperadas en Argentina, Buenos Aires, Cooperativa Chilavert.

RUGGERI, Andrés, WERTHEIMER, Marina; GALEAZZI, Cecilia; y GARCÍA, Fernando (2012): Autogestión y cooperativismo, Universidad de Buenos Aires, Facultad de Filosofía y Letras. En: http://www.recuperadasdoc.com.ar/cuadernos_autogestion. html

SASIA, Pedro M. y DE LA CRUZ, Cristina (2008): Banca ética y ciudadanía, Madrid, Trotta.

SARASUA, Jon, y UDAONDO, Ainara (2004): Autogestión y globalidad: Situar la autogestión económica en el mundo actual, Mondragón, Lanki Ikertegia (Mondragón Unibertsitatea).

SHAW, Eleanor, and CARTER, Sara (2007): «Social entrepreneurship: Theoretical antecedents and empirical analysis of entrepreneurial processes and outcomes», Journal of small business and enterprise development, 14(3), 418-434.

SPEAR, Roger (2006): «Social entrepreneurship: a different model?», International journal of social economics, 33(5/6), 399-410.

UTTING, Peter (ed) (2015): Social and Solidarity Economy Beyond the Fringe?, London, Zed Books.

VECIANA, Paula (2007): Las empresas de inserción en España, Barcelona, Fundació Un Sol Món de Caixa de Catalunya.

VIETA, Marcelo (2010): «The New Cooperativism», Affinities: A Journal of Radical Theory, Culture, and Action, 4(1). 